\title{
The Crab pulsar and its red knot in the near-infrared ${ }^{\star}$
}

\author{
J. Sollerman ${ }^{\star \star}$ \\ Stockholm Observatory, AlbaNova, 10691 Stockholm, Sweden \\ Received 28 January 2003 / Accepted 22 April 2003

\begin{abstract}
We present near-infrared observations obtained with ISAAC on the VLT of the Crab pulsar and its environment. Photometry of the pulsar in $J_{\mathrm{s}}, H$ and $K_{\mathrm{s}}$ shows the pulsar spectrum to extend fairly smoothly from the UV/optical regime. PSF subtraction of the pulsar allows us to study its immediate neighborhood in some detail. In particular, the knot positioned just 0.' 6 from the pulsar has been revealed in the IR. Using also archival HST data for the knot, we have measured its broad band spectrum to rise steeply into the IR, in contrast to the spectrum of the pulsar itself.
\end{abstract}

Key words. pulsars: individual: PSR B0531+21 - ISM: supernova remnants - ISM: individual objects: Crab Nebula

\section{Introduction}

The Crab nebula is the remnant of a supernova that exploded in $1054 \mathrm{AD}$ (see e.g., Sollerman et al. 2001 and references therein). At the heart of the nebula resides the Crab pulsar, the $m_{V} \sim 16$ object that powers the entire visible nebula. Although the Crab nebula and its pulsar are among the most studied objects in the sky, this astrophysical laboratory still holds many secrets about how supernovae explode and about how pulsars radiate and energize their surrounding nebulae.

A main theme for pulsar research has been to understand the emission mechanism for the non-thermal pulsar radiation. This is still to be accomplished. No comprehensive model exists that can explain all the observed features of the radiation. Observationally, only recently was a broad range UV-optical spectrum of the Crab pulsar published (Sollerman et al. 2000). Here we have extended this study of the pulsar spectral energy distribution into the near-infrared (IR).

Even if most of the research on the Crab pulsar has concerned the radiation mechanism, almost all of the spin-down energy comes out in the particle wind. The stunning image of the pulsar environment obtained with Chandra captures a glimpse of the energetic processes at work (Weisskopf et al. 2000).

Direct evidence of the pulsar activity has long been seen in the system of moving synchrotron wisps close to the pulsar itself. The detailed study of the wisps was initiated by Scargle (1969), and is now conducted at higher resolution using the HST (Hester et al. 1995; Hester et al. 2002).

Perhaps the most astounding discovery in these HST images was the knot situated merely 0,' 6 from the pulsar. At $2 \mathrm{kpc}$ this amounts to a projected distance of only $1000 \mathrm{AU}$.

* Based on observations collected at the European Southern Observatory, Paranal, Chile (ESO Programme 66.D-0251).

$\star \star$ e-mail: jesper@astro.su.se
Hester et al. (1995) interpreted this feature as a shock in the pulsar polar wind. Our IR observations allow us to further investigate these manifestations of the magnetic relativistic wind from the pulsar.

\section{IR photometry, reductions and results}

\subsection{Observations and basic reductions}

We obtained IR imaging in the short wavelength (SW) mode of ISAAC $^{1}$ at UT1 of VLT on October 13, 2000. ISAAC (Moorwood et al. 1999) is equipped with a $1024 \times 1024$ pixel Rockwell Hawaii array providing a plate scale of $0 .{ }^{\prime} 147$ pixel $^{-1}$ and a total field of view of about $2.5 \times 2.5$. The exposures were obtained in $J_{\mathrm{s}}, H$ and $K_{\mathrm{s}}$. The $J_{\mathrm{s}}$ filter is a narrower version of the classical $J$ band filter. It is centered on $1.24 \mu \mathrm{m}$ with a width of $0.16 \mu \mathrm{m}$, and is constructed to deliver more accurate photometry due to a significantly lower background without any real loss in efficiency (e.g., Simons \& Tokunaga 2002). As the pulsar is a relatively bright source, short exposure times were used. In all cases the observations were obtained using a detector integration time of $2.0 \mathrm{~s}$. For each position $13 \mathrm{such}$ exposures were stacked before readout, and we obtained images at 6 positions with the telescope being offset by random amounts up to 25 arcsec between the individual images. The total exposure time was thus $156 \mathrm{~s}$ per band. The excellent image quality provided by ISAAC also allowed a detailed view of the central region of the Crab nebula. The images are displayed in Fig. 1.

The data were reduced using both IRAF and the JITTER routine within the ECLIPSE package. This includes bias subtraction, flat fielding, sky subtraction and combination of the

\footnotetext{
${ }^{1}$ Infrared Spectrometer And Array Camera, see http://www.eso.org/instruments/isaac/ for details.
} 

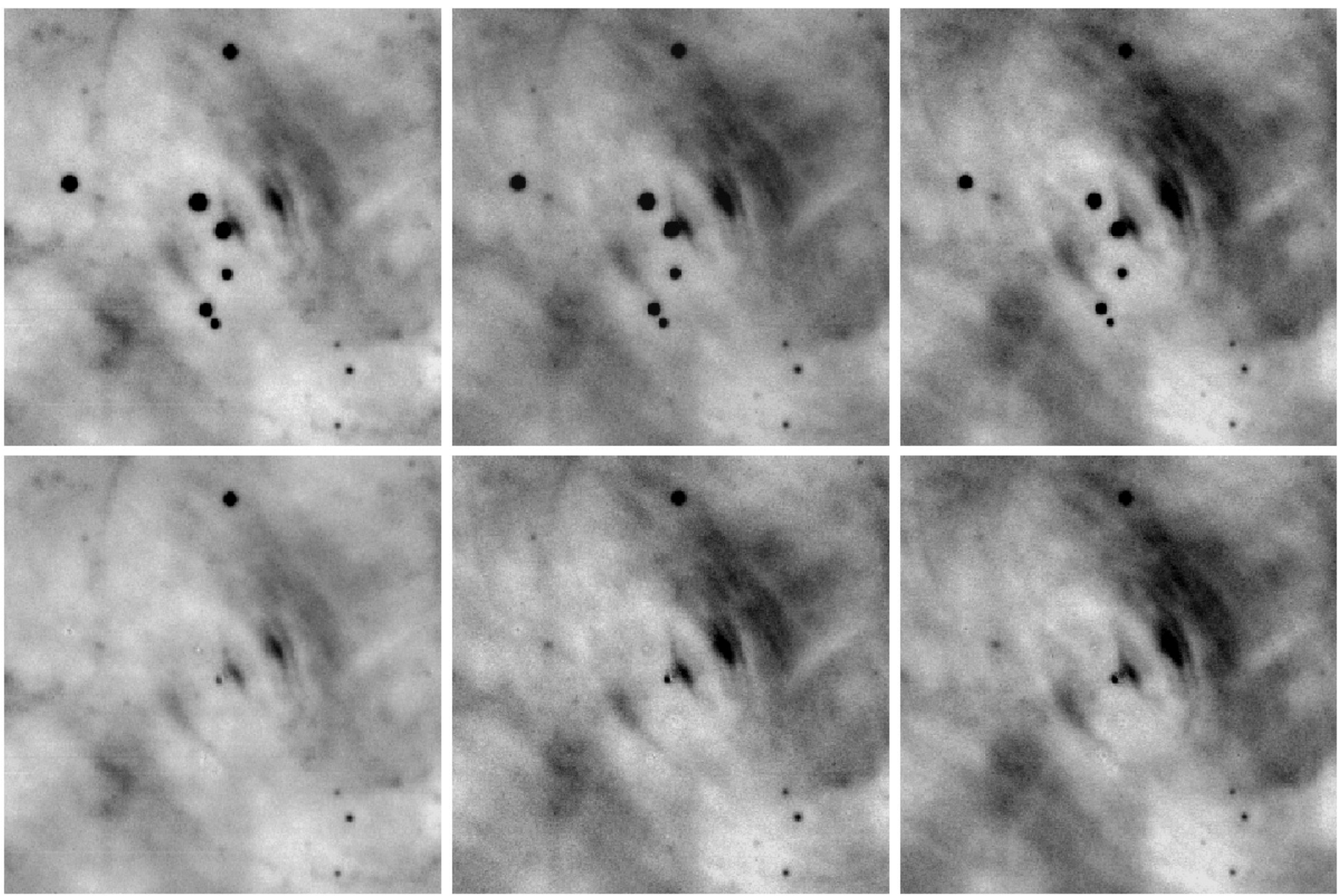

Fig. 1. The central part of the Crab nebula in the infrared, $J_{\mathrm{s}}$ (left), $H$ (middle) and $K_{\mathrm{s}}$ (right). Observations obtained with ISAAC on 13 October 2000. The pulsar is the lower right (South Preceding) of the two bright objects near the center of the field. The field of view shown is $60^{\prime \prime} \times 60^{\prime \prime}$ centered on the pulsar. North is up and East to the left. The upper panels show the original images, and the lower panels show the images where several objects, including the pulsar, have been PSF subtracted. The lower panels are intended to reveal the knot positioned very close to the pulsar. Detailed color images of these frames are presented in Sollerman \& Flyckt (2002).

different exposures. We found no significant difference in the photometry obtained from these reductions packages.

The photometry was achieved in the following way. Photometric standard stars are observed regularly with ISAAC in service mode. On October 13 the stars S889-E and S121-E (Persson et al. 1998) were observed in $J, J_{\mathrm{s}}, H$ and $K_{\mathrm{s}}$. S121-E was observed immediately before the Crab observations. We reduced these images and measured the flux of the standard stars to establish zero-points in all filter bands. This night had very low humidity and the seeing was typically just under $0 .{ }^{\prime \prime} 8$. The FWHM varies from $\sim 0$ ' $^{\prime} 88-0{ }^{\prime} 65$. in the final $J_{\mathrm{s}}$ and $K_{\mathrm{s}}$ images, respectively. No atmospheric extinction corrections were applied, as they are smaller than $0.01 \mathrm{mag}$.

\subsection{Photometry}

Photometry was obtained of the Crab pulsar and some of the stars in the field using point-spread function (PSF) fitting. A PSF was constructed using bright and relatively isolated stars in the frame. This PSF was then subtracted from the image of the pulsar and some nearby stars using ALLSTAR within the DAOPHOT package (Stetson 1987). This improves the accuracy in this crowded region, in particular for the pulsar which has a complex surrounding. The magnitudes were aperture corrected using bright isolated standard stars. The aperture corrections amounted to about $0.1 \mathrm{mag}$. For the three isolated aperture stars we measure no significant offset between our photometry and the photometry in the 2MASS catalogue. They agree to within 0.05 mag. For the more crowded and fainter stars we regard our photometry to be superior, due to the excellent seeing and to the performed PSF subtraction. This is particularly true for the Crab pulsar. The magnitudes for the Crab pulsar, and several of its nearby stars, are presented in Table 1.

The formal errors for the pulsar and for the nearby stars from DAOPHOT are less than $0.01 \mathrm{mag}$. The uncertainties in the standard star magnitudes are also much below $0.01 \mathrm{mag}$, and the zeropoints obtained for the two standards agreed to within $0.01 \mathrm{mag}$ in all bands. The total error is instead likely to be dominated by small errors in the PSF subtraction and in the aperture corrections. We estimate a conservative error budget of $0.05 \mathrm{mag}$ for the pulsar and the nearby stars in the three bands observed.

Using the conversions from Wilson et al. (1972) we have converted the magnitudes to fluxes. Those were then 


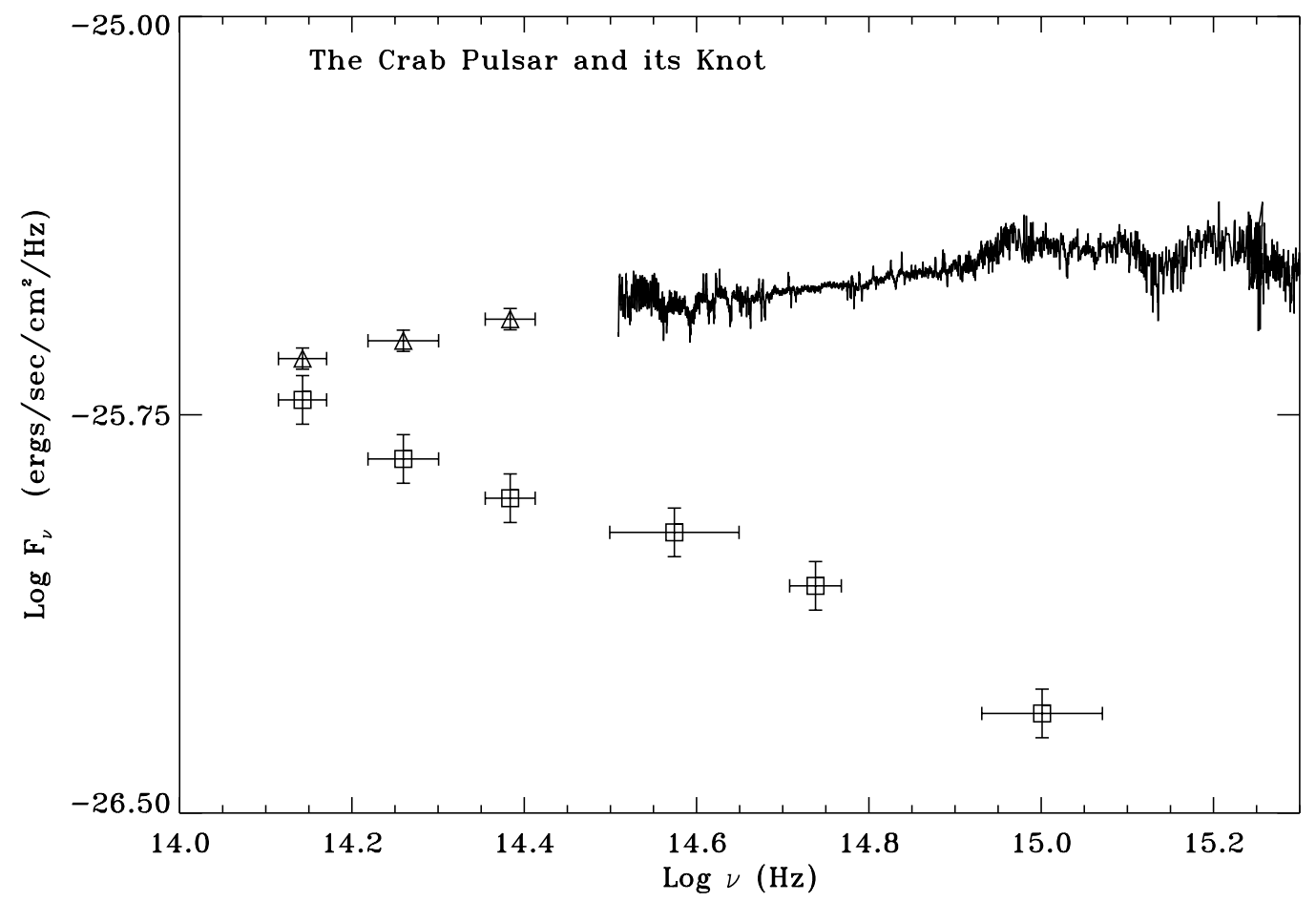

Fig. 2. Spectral energy distribution of the Crab pulsar. The optical and UV data are from Sollerman et al. (2000). The triangles are our new ISAAC measurements. Also shown (squares) are the fluxes of the knot, here multiplied by a factor ten. The optical data for the knot is from HST. The knot data error bars illustrate an uncertainty of $10 \%$ in the flux. All observed fluxes were dereddened using $R=3.1$ and $E(B-V)=0.52$.

Table 1. $J_{\mathrm{s}}, H$ and $K_{\mathrm{s}}$ photometry of the Crab pulsar and its nearest neighbors ${ }^{a}$.

\begin{tabular}{lccc}
\hline \hline $\begin{array}{l}\text { Offsets from Pulsar } \\
\text { (arcsec) }\end{array}$ & $J_{\mathrm{s}}$ & $H$ & $K_{\mathrm{s}}$ \\
\hline Crab Pulsar & 14.80 & 14.27 & 13.77 \\
$6.29 \mathrm{~N}, 20.37 \mathrm{E}$ & 14.64 & 14.26 & 14.14 \\
$3.82 \mathrm{~N}, 3.24 \mathrm{E}$ & 14.08 & 13.75 & 13.65 \\
$5.84 \mathrm{~S}, 0.51 \mathrm{~W}$ & 16.14 & 15.41 & 15.24 \\
$10.58 \mathrm{~S}, 2.29 \mathrm{E}$ & 15.29 & 14.90 & 14.80 \\
$12.43 \mathrm{~S}, 1.05 \mathrm{E}$ & 16.50 & 16.11 & 16.06 \\
\hline
\end{tabular}

${ }^{a}$ The errors are $\lesssim 0.05 \mathrm{mag}$ in all bands.

dereddened using $E(B-V)=0.52$ (Sollerman et al. 2000), and the extinction law from Fitzpatrick (1999). The results are plotted in Fig. 2, which also includes the optical-UV data from Sollerman et al. (2000).

We note that our IR magnitudes deviate from the recent results in the 2MASS point source catalogue. Our measurements give a pulsar that is fainter by $0.32,0.35$, and 0.27 mag in $J_{\mathrm{s}}$, $H$ and $K_{\mathrm{s}}$, respectively. The 2MASS magnitudes are consistent with the data from Eikenberry et al. (1997) (to within $0.1 \mathrm{mag}$ ), but since our relatively isolated standards in the field show good agreement with 2MASS we do not think this is due to an offset in the zero-point.

Our ISAAC photometry has excellent signal and image quality. In the crowded region around the pulsar, this significantly improves the background subtraction. In particular, PSF subtraction excludes contributions from wisps and the nearby knot (Fig. 1). The accuracy of the PSF fit can be seen in the subtracted images, where the knot is clearly revealed. We believe that the difference in magnitudes is due to our ability to better separate the background from the pulsar emission.

We note that our measurements show a good agreement with previous measurements using time-resolved photometry of the Crab (Penny 1982; Ransom et al. 1994), although the errors claimed by Ransom et al. are rather large. Such measurements generally use a large aperture and simply assume any non-varying contribution to be due to the background. By integrating under the pulsar light curve, they measure the pulsating contribution of the pulsar flux. We believe that with the good image quality of our data, we are now able to subtract virtually all background from the pulsar contribution. The implications of the pulsar photometry are discussed below.

\subsection{The IR images}

Our new images show most of the well known features of the inner part of the Crab Nebula. In particular, the wisps are clearly visible in higher detail than previously obtained in the IR. Some filaments are also seen, most strongly in the $J_{\mathrm{s}}$ band. This is most likely due to emission from [Fe II] $1.26 \mu \mathrm{m}$. The [Fe II] $1.64 \mu \mathrm{m}$ line in the $H$-band was detected by Graham et al. (1990). It appears less conspicuous, probably also because the narrower $J_{\mathrm{s}}$ filter includes less continuum. Finally, the $K_{\mathrm{s}}$ band image is clearly dominated by amorphous synchrotron emission. With suitable cuts the pulsar image appears slightly elongated. This is due to the presence of the knot first identified by Hester et al. (1995) on HST/WFPC2 images. To reveal this structure in our images we constructed a PSF of 
suitable stars in the image, and subtracted this from the image of the pulsar and some nearby stars. In the subtracted images, the knot is clearly visible in all three bands (Fig. 1).

From our $J_{\mathrm{s}}, H$ and $K_{\mathrm{s}}$ band images it is possible to create a color image. This was done by Sollerman \& Flyckt (2002), who also presented a color image made out of the PSF subtracted frames. In the latter image, it can be seen that the knot is redder than, e.g., the wisps.

To quantify this color difference we have estimated the magnitudes of the knot as well as of the nearby wisp 1 (Scargle 1969). The knot was measured within an aperture of $0 .{ }^{\prime \prime} 9$ and the wisp was also simply measured with a circular aperture with a radius of 1. ". 2 . The spectral energy distribution of the knot is shown in Fig. 2. It is clearly red. In the $K_{\mathrm{s}}$-band the flux from the knot amounts to about $8 \%$ of the flux of the pulsar. The stationary wisp appears to have a flatter spectrum in this regime, as does the pulsar itself (see below).

\section{Optical data from the Hubble Space Telescope}

Data on the Crab pulsar are also available in the HST archive. Most of the observations are from the comprehensive monitoring programme of the inner parts of the nebula (P.I. J. Hester). These frames allow a detailed study of both the spectral and temporal properties of the knot. In August 1995 the region was observed in three filters (F300W, F574M, F814W). We performed aperture photometry of the knot at these images. An aperture with a radius of 7 pixels $\left(0.0^{\prime \prime} 32\right)$ was used, and the background was measured in an equal size aperture positioned at the opposite side of the pulsar. The magnitudes were converted to dereddened fluxes and are plotted in Fig. 2. The uncertainties in these fluxes are not negligible. This is because the pulsar is saturated in all images, and no accurate PSF subtraction of the pulsar could be made. The absolute flux of the knot also depends on the chosen aperture size. However, to estimate a spectral energy distribution we measured the knot with the same aperture size in the three filter images. Then the formal errors due to photon statistics is $\sim 5 \%$, but the position of the region where we measure the background can introduce uncertainties of up to $\sim 10 \%$. In Fig. 2 we have included error bars of $10 \%$ in the flux for all knot measurements, and also indicated the widths of the used filters. Despite these uncertainties, the color trend found is unambiguous and confirms the results from our IR measurements. We measured the dereddened spectral index for the knot to be $\alpha_{v} \sim-0.8$ in the HST bands $\left(F_{v} \propto v^{\alpha_{v}}\right)$ in full agreement with the slope determined from the IR data (Fig. 2). The spectral energy distribution of the wisp was also estimated in both the HST and ISAAC images, and the slope was found to be negative $\left(\alpha_{v}<0\right)$ although substantially flatter than for the knot. The knot is indeed redder than the surrounding nebula, and substantially redder than the pulsar itself.

The wealth of data in the F574M filter available in the archive also allows a study of the temporal behavior. The knot is present in all frames, and thus appears quasistationary for more than six years, although the position appears to vary at the $0{ }^{\prime} 1$ level. The dereddened flux of the knot within the aperture is measured to be $\sim 9 \times 10^{-28} \mathrm{ergs} \mathrm{s}^{-1} \mathrm{~cm}^{-2} \mathrm{~Hz}^{-1}$ in August 1995 , but variations of the flux by at least $50 \%$ are observed. This means that the rather smooth connection between the optical and IR data for the knot in Fig. 2 may be partially superficial. However, we have also checked the F547M data from 1995 against HST data taken the same day as our ISAAC data. We measured only a small increase $(\sim 10 \%)$ in the flux of the knot between the 1995 image and the image obtained in 2000. This is likely to be within the errors of our measurements and no correction for this evolution has been applied to Fig. 2 .

\section{Discussion and implications}

\subsection{The pulsar}

We have reported magnitudes for the Crab pulsar in the IR, where the observational situation has previously been rather uncertain (compare, e.g., estimates by Penny 1982; Middleditch et al. 1983; Eikenberry et al. 1997). Together with recent optical-UV data this significantly revises the observational basis for the pulsar emission mechanism. The slope in the near-IR is somewhat steeper than in the UV/optical region. A power-law fit to the three near-IR points gives a slope of $\alpha_{v}=0.31 \pm 0.02$, whereas the UV/optical spectral index is $\alpha_{v}=0.11$ (Sollerman et al. 2000).

A spectral index of one third is expected if the nonthermal emission is due to synchrotron radiation of relativistic particles in the magnetosphere of the pulsar. For a monochromatic particle distribution a spectral flux of $F_{v} \propto v^{1 / 3}$ is expected in the low frequency range below some maximum frequency $v_{\mathrm{m}}$ (e.g., Ginzburg \& Syrovatskii 1965).

Models for the optical emission from the Crab pulsar based on the synchrotron mechanism often aim at reproducing the maximum frequency in the optical range $\left(v_{\mathrm{m}} \sim 5 \times 10^{14} \mathrm{~Hz}\right.$, eg., Stoneham 1981; Mavlov \& Machabeli 2001; Lyne \& Graham-Smith 1998). This is mainly based on the observations by Oke (1969), and is not supported by the more recent observations presented in Sollerman et al. (2000). A model in which the flat spectrum extending all the way into the $\mathrm{UV}$ is due to synchrotron emission at very small pitch angles (Crusius-Wätzel et al. 2001) assumes a very rapid spectral decline towards infrared frequencies. This has been reported by Middleditch et al. (1983) and is sometimes interpreted as synchrotron self-absorption (see Lyne \& Graham-Smith 1998 and references therein). The near-IR data presented here do not support such a steep decline, rather a possible smooth leveling of the spectral index from UV via the optical and into the nearIR. If the $L$-band measurement of Penny (1982) is correct, as seems to be the case for his $J, H$, and $K$ band data, there is no evidence for a sharp decrease into the $L$-band. New observations are underway to investigate the IR spectral shape (see below). These new data call for a fresh look on the emission mechanism scenarios for young pulsars.

\subsection{The knot}

For the knot, we have shown that its structure is quasistationary, and that its emission has a red spectrum. Most quantitative modeling for the overall properties of the Crab nebula is based on the theory outlined by Rees \& Gunn (1974) and 
extended by Kennel \& Coroniti (1984). However, few papers address the properties of the knot. Lou (1998) presented a formation scenario in terms of MHD theory, while Shapakidze \& Machabeli (1999) argue for a plasma mechanism. None of these scenarios predict a very red spectral distribution.

Another area where caution may be required is in the recent claims of weak off-pulse emission from the Crab pulsar in the visible (Golden et al. 2000). It is clear that a knot close to the pulsar has to be seriously considered in these kinds of studies. The off-pulse emission detected by Golden et al. (2000) could either be from the pulsar itself, or from a nearby knot. The strong polarization and in particular the red spectrum of the unpulsed emission, in contrast to the pulsed pulsar emission, is clearly consistent with a knot. The knot we have detected in the IR is $0 . ' 6$ from the pulsar and could probably be excluded if the seeing is good enough and the neighboring star is used as a PSF star, but more nearby features may also be present. It is also unclear if the knot $0 .^{\prime \prime} 6$ from the pulsar is luminous enough to explain the off-pulse emission discussed in Golden et al. (2000; Shearer \& Golden 2002), although the intensity of the knot is clearly varying (see also Hester et al. 2002). This at least calls for caution in the interpretation of the off-pulse data.

\subsection{Other pulsars}

Although the Crab pulsar is the brightest of the optical pulsars, a few more pulsars have now been detected in the near-IR. A comparison is made in Fig. 3, adapted from Shibanov et al. (2003). It is clear that none of these pulsars show a low-energy break. In particular, the previous indication of a break in the Vela pulsar spectrum at $6500 \AA$ (Nasuti et al. 1997) is not supported by these new observations. The Vela pulsar spectrum is rather flat over the entire optical/IR regime. Using the best available data; ground-based $U B$ photometry from Nasuti et al. (1997), HST F555W data from Mignani \& Caraveo (2001), and HST F675W and F814W as well as VLT/ISAAC $J_{\mathrm{s}}$ and $H$ data from Shibanov et al. (2003), we can fit a power law to the entire optical/IR regime. The amount of extinction is somewhat uncertain, affecting the derived spectral index. Including the errorbars in the fit gives $\alpha_{v} \sim-0.1$ for $A_{V}=0.18$ and $\alpha_{v} \sim+0.1$ for $A_{V}=0.4$.

As seen in Fig. 3, the middle-aged pulsars Geminga and PSR $0656+14$ also show a fairly flat spectrum in this range. The optical emission can, however, also be interpreted as a combination of two components; a thermal component and a steeper non-thermal power-law component which is increasing into the IR (e.g., Pavlov et al. 1997; Martin et al. 1998). This has been interpreted in terms of spectral evolution with pulsar age (e.g., Koptsevich et al. 2001).

In this paper we have demonstrated that accurate measurements of the magnitudes of the Crab pulsar requires good signal-to-noise and spatial resolution. It is clear that the uncertainties in measuring much fainter pulsars can be considerable. It is important to keep these uncertainties in mind when interpreting the scarce datasets available for optical pulsars. Many claims in the literature regarding flux excesses or dips in the

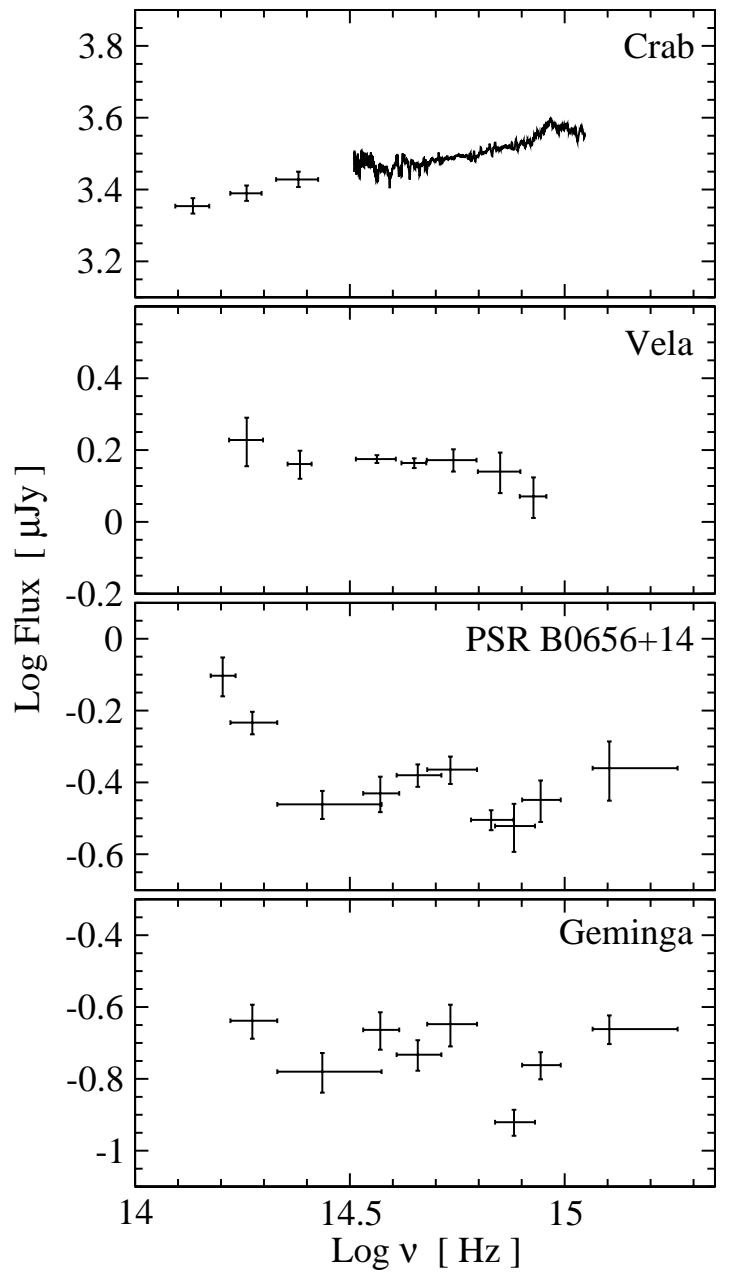

Fig. 3. Comparison between the pulsars with observed near-IR emission, from Shibanov et al. (2003). See text for discussion.

spectral energy distribution of optical pulsars appears to have little significance.

Another interesting result by Shibanov et al. (2003) is the tentative near-IR detection of an extended structure around the Vela pulsar. This could be an IR counterpart to the X-ray nebula established by Chandra observations (Helfand et al. 2001). As these structures are not detected in the optical, the spectra must be very red, in some way resembling the red structures found in the Crab pulsar neighborhood.

\section{Future plans}

The Crab pulsar and its environment continue to be the prime astrophysical laboratory for the study of the pulsar emission mechanism and the spin-down powering of pulsar nebulae. Although much observational effort has been put into this object, a modern re-investigation is likely to clean up the many contradictory measurements. A project to acquire optical imaging in good seeing is underway to determine the knot-subtracted spectral energy distribution of the pulsar. We will also pursue IR observations using the NAOS/CONICA instrument on VLT to monitor the structures close to the pulsar in even better spatial resolution. These observations will also 
extend into the $L$-band to clarify if the knot contributes significantly to the emission at these frequencies, and establish whether or not the IR drop of the pulsar is real.

Acknowledgements. Special thanks to Veronica Flyckt who did a substantial part of the work on this project during her master thesis conducted at ESO Garching. Also thanks to Yuri Shibanov and Peter Lundqvist for comments on the manuscript, and to Bruno Leibundgut for comments and for support from ESO Office of Science during Veronicas stay in Garching.

\section{References}

Crusius-Wätzel, A. R., Kunzl, T., \& Lesch, H. 2001, ApJ, 546, 401

Eikenberry, S. S., Fazio, G. G., Ransom, S. M., et al. 1997, ApJ, 477, 465

Fitzpatrick, E. L. 1999, PASP, 111, 63

Ginzburg, V. L., \& Syrovatskii, S. I. 1965, ARA\&A, 2, 297

Golden, A., Shearer, A., \& Beskin, G. M. 2000, ApJ, 535, 373

Graham, J. R., Wright, G. S., \& Longmore, A. J. 1990, ApJ, 352, 172

Helfand, D. J., Gotthelf, E. V., \& Halpern, J. P. 2001, ApJ, 556, 380

Hester, J. J., Scowen, P. A., Sankrit, R., et al. 1995, ApJ, 448, 240

Hester, J. J., Mori, K., Burrows, D., et al. 2002, ApJ, 577, L49

Kennel, C. F., \& Coroniti, F. V. 1984, ApJ, 283, 710

Koptsevich, A. B., Pavlov, G. G., Zharikov, S. V., et al. 2001, A\&A, 370,1004

Lou, Y.-Q. 1998, MNRAS, 294, 443

Lyne, A. G., \& Graham-Smith, F. 1998, Pulsar Astronomy (Cambridge: Cambridge Univ. Press)

Martin, C., Halpern, J. P., \& Schiminovich, D. 1998, ApJ, 494, 211

Mavlov, I. F., \& Machabeli, G. Z. 2001, ApJ, 554, 587
Middleditch, J., Pennypacker, C., \& Burns, M. S. 1983, ApJ, 273, 261

Mignani, R. P., \& Caraveo, P. A. 2001, A\&A, 376, 213

Moorwood, A., Cuby, J.-G., Ballaster, P., et al. 1999, Messenger, 95, 1

Nasuti, F. P., Mignani, R., Caraveo, R. P., \& Bignami, G. F. 1997, A\&A, 323, 839

Oke, J. B. 1969, ApJ, 158, 90

Pavlov, G. G., Welty, A. D., \& Cordova, F. A. 1997, ApJ, 489, 75

Penny, A. J. 1982, MNRAS, 198, 773

Persson, S. E., Murphy, D. C., Krzeminski, W., Roth, M., \& Rieke, M. J. 1998, AJ, 116, 2475

Ransom, S. M., Fazio, G. G., Eikenberry, S. S., et al. 1994, ApJ, 431, 43

Rees, M. J., \& Gunn, R. E. 1974, MNRAS, 167, 1

Scargle, J. D. 1969, ApJ, 156, 401

Shapakidze, D., \& Machabeli, M. 1999, Ptep. Proc, 371

Shearer, A., \& Golden, A. 2002, in Neutron stars, Pulsars and Supernova remnants, ed. W. Becker, H. Lesch, \& W. Trümper, MPE Rep. 278, 44

Shibanov, Y. A., Koptsevich, A. B., Sollerman, J., \& Lundqvist, P. 2003, A\&A, 406, 645

Simons, D. A., \& Tokunaga, A. 2002, PASP, 114, 69

Sollerman, J., Lundqvist, P., Lindler, D., et al. 2000, ApJ, 537, 861

Sollerman, J., Kozma, C., \& Lundqvist, P. 2001, A\&A, 366, 197

Sollerman, J., \& Flyckt, V. 2002, Messenger, 107, 32

Stetson, P. B. 1987, PASP, 99, 191

Stoneham, R. J. 1981, MNRAS, 199, 219

Weisskopf, M. C., Hester, J. J. Tennant, A. F., et al. 2000, ApJ, 536, L81

Wilson, W. J., Schwartz, P. R., Neugebauer, G., Harvey, P. M., \& Becklin, E. E. 1972, ApJ, 177, 523 